\title{
Toward comparability of anti-drug antibody assays: is the amount of anti-drug antibody-reagent complexes at cut-point (CP-ARC) the missing piece?
}

\author{
Gregor Jordan ${ }^{1} \&$ Roland F Staack*,1 \\ ${ }^{1}$ Roche Pharma Research \& Early Development (pRED), Pharmaceutical Sciences, Bioanalytical R\&D, Roche Innovation Center \\ Munich, Roche Diagnostics GmbH, Nonnenwald 2, 82377 Penzberg, Germany \\ *Author for correspondence: Tel.: +49 885660 4348; roland.staack@roche.com
}

Immunogenicity testing is a mandatory and critical activity during the development of therapeutic proteins. Multiple regulatory guidelines provide clear recommendations on appropriate immunogenicity testing strategies and required bioanalytical assay performances. Unfortunately, it is still generally accepted that a comparison of the immunogenicity of different compounds is not possible due to apparent performance differences of the used bioanalytical methods. In this perspective, we propose the 'cut-point anti-drug antibody-reagents complex' (CP-ARC) concept for technical comparability of the bioanalytical methods. The feasibility and implementation in routine assay development is discussed as well as the potential improvement of reporting of bioanalytical immunogenicity data to allow comparison across drugs. Scientific sound comparability of the bioanalytical methods is the first step toward comparability of clinical immunogenicity.

First draft submitted: 19 May 2020; Accepted for publication: 17 June 2020; Published online: 7 July 2020

Keywords: ADA assay $\bullet$ anti-drug antibodies $\bullet$ comparability $\bullet$ cut-point $\bullet$ drug tolerance $\bullet$ immunogenicity

One of the major threats of a successful treatment of patients with a therapeutic protein is the occurrence of an immune response, since it might lead to loss of exposure and efficacy or could trigger unwanted safety events [1-3]. For that reason, immunogenicity testing is a crucial part of the clinical development of therapeutic proteins. Multiple regulatory guidelines are available which provide recommendations on how state-of-the-art immunogenicity testing shall be performed $[4,5]$. Immunogenicity testing is performed by screening for and characterization of potentially formed anti-drug antibodies (ADAs). Typically, bridging format ligand-binding assays (LBAs) are used for screening, confirmation and for titer determination in a three-tiered approach, after determination of an assay cut-point based on a statistical evaluation of blank sample signals [4-7]. The information on the immunogenicity of an individual drug candidate is not only a regulatory requirement but also is very important for company internal decision making as well as for decision making of the regulators and for physicians. The process of evaluating the immunogenicity of an individual therapeutic protein drug candidate is very well established. The comparability of the immunogenicity of different compounds, however, is deemed highly difficult due to differences in the applied methodologies/assays $[4,5,8,9]$. This is a particular challenge for the comparison of the immunogenicity of a biosimilar drug to the originator drug [10-12].

It is generally accepted that immunogenicity, particularly ADA incidences, of different therapeutic proteins cannot be compared, particularly due to differences in assay performances of the applied ADA methods. The major limitations that are held responsible for the 'non-comparability' of ADA assays are apparent performance differences in respect of sensitivity and drug tolerance. The sensitivity of an assay is determined by evaluating the concentration of an ADA-positive control antibody, typically animal-derived immunopurified polyclonal or monoclonal antibody preparations that is detectable at the defined assay cut-point. The apparent assay performance parameters especially assay sensitivity and also drug tolerance, depend on the used ADA-positive control [13], which can have different binding properties. There are a few studies reporting the impact of differences in ADA affinity on assay sensitivity 
on different technology platforms [7,14-18]. The difficulties in understanding and interpretation of assay results is discussed in the scientific community [19], most notably the potential overestimation of the sensitivity due to use of high affinity ADA-positive controls $[4,5]$. There are no specific guidelines available on the development and selection of a polyclonal or monoclonal positive control antibody nor is any 'standard' positive control possible that could be used across assays; the selection of the positive control antibody is done at the discretion of the assay developer.

A comparison of the immunogenicity of different therapeutic proteins, however, would be a highly desirable goal. This would help to compare different drug candidate compounds during drug development, would enable an appropriate immunogenicity comparison of biosimilars to its originators and finally, would enable the selection of the most suited treatment option in clinical practice. Such a comparison certainly requires a holistic view on immunogenicity, PK, PD and safety data. The first step for such an evaluation is the possibility to compare immunogenicity data generated by different assays. To achieve this goal, the US FDA discussed the need of a 'universally ADA-positive control' [5]. The availability of such a control antibody would be the ultimate solution, however, as mentioned above, it is rather unlikely that such a compound will ever be available, since it needs to be drug specific and, with the increased number of new and complex therapeutic protein drugs beyond therapeutic monoclonal antibodies [20], should enable comparability between different therapeutic compound classes.

The responsibility to provide the required data lies within the bioanalytical community. The first, and maybe even the most critical step toward a comparability of immunogenicity, is the comparability of the technical performance of the used ADA assays. The bioanalytical community should take this highly important and scientifically interesting task and should jointly try to find solutions to improve the current situation with the ultimate goal to enable a scientifically sound comparison of immunogenicity of different compounds.

The first step toward this goal would be a better understanding of the true assay performance parameters. The critical role of the binding kinetic properties of the used ADA-positive control for assay development and validation is well understood [7,8,14-17]. Egging et al. performed a detailed investigation into the correlation of apparent assay sensitivity and drug tolerance in relation to the binding properties of the ADA-positive control antibody preparation [14]. However, in contrast to the theory that this is the key to understand ADA assay performance, no relation between affinity of the positive control and sensitivity or drug tolerance was found in this study.

Our hypothesis, why this evaluation did not confirm the theory and did not show the expected relation between affinity of the ADA-positive control and assay performance parameters is, that the important role of the assay reagents and conditions, which are additional binding partners in the complex binding interaction of a bridging LBA, was not considered.

In this perspective the 'cut-point anti-drug antibody reagents complex' (CP-ARC) concept, our proposal for technical comparability of ADA assays, is presented. The concept is introduced; its potential, technical feasibility and implementation in routine bioanalytical drug development support as well as the current challenges are discussed and illustrated by an example, focusing on the one-step bridging assays, the standard format for immunogenicity testing.

\section{Is the amount of cut-point anti-drug antibody-reagent - complexes the key assay performance parameter?}

When discussing the ADA assay performance features, we should critically question our current thinking. The scientific community generally agrees that the assay sensitivity is not dependent on the used ADA-positive control. However the question is, whether we actually fully exploit the available information on the used positive control to improve our understanding? How difficult is it to generate the missing information to enable a true comparison between assays?

Immunogenicity testing is commonly performed by LBAs. As for all LBAs, the detection of an analyte is achieved by binding of the assay reagents to the analyte to form immune complexes that are finally detected (e.g., by a colorimetric reaction, fluorescence, electrochemiluminescence, etc). Consequently, the most critical step is the binding event of the analyte and the reagents, as emphasized in the name 'ligand-binding assay'.

For most LBA assays that detect one specific analyte (e.g., assays for drug quantification), there are clearly defined binding events that are important for the assay performance, namely the binding of the capture and detection reagents to the analyte. Though the characterization of the binding kinetics of a reagent toward the analyte is not a significant technical hurdle and even an important and critical part of the reagent development and characterization process [21-24] it is either not consequently performed by assay developers or not consequently used to assess the 


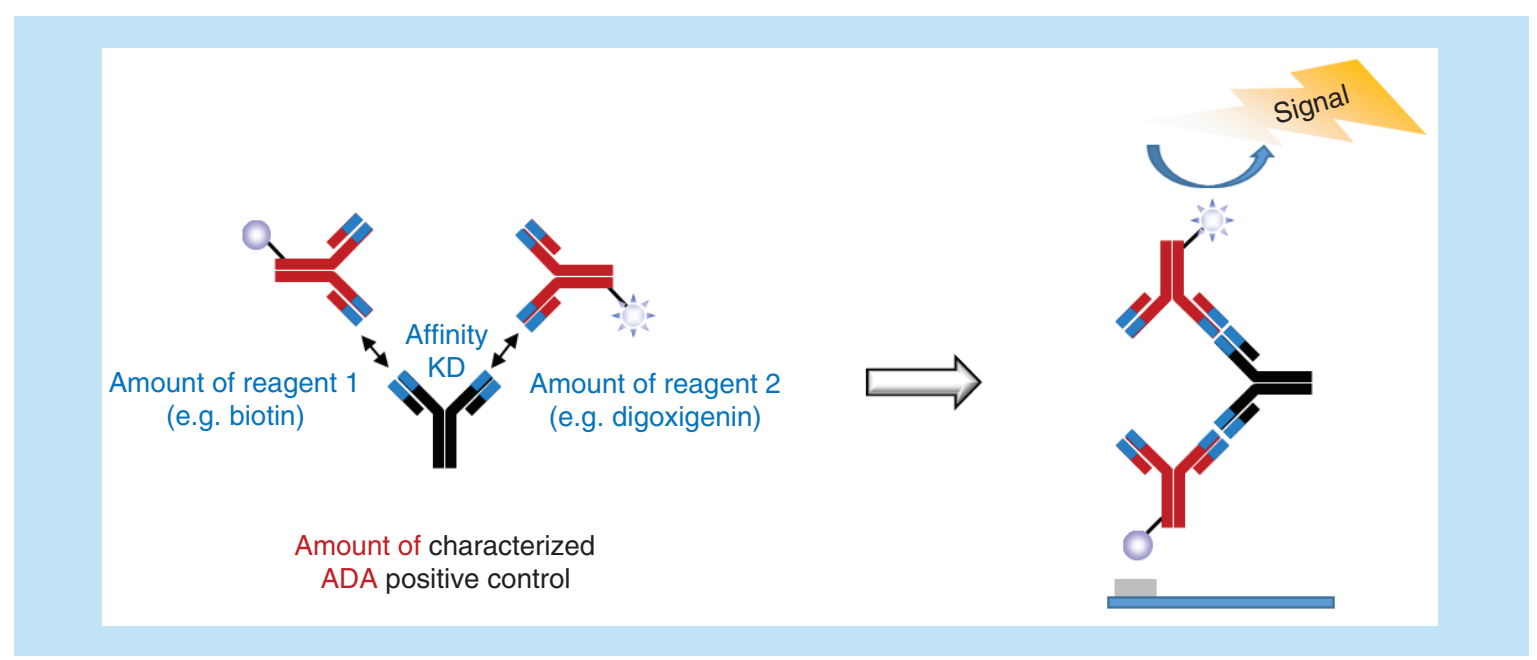

Figure 1. Anti-drug antibody-reagent complexes. The signal generated in an LBA assay and consequently the sensitivity depends on the formation of antibody reagent complexes. The amount of these important complexes is dependent on the amount of the ADA (positive control), the amount and ratio of the capture and detection reagents and the affinity between the ADA-positive control and the reagents.

ADA: Anti-drug antibody.

performance parameters of an assay. Since the binding events during the analysis of calibrators and samples are identical (at well-defined, known assay conditions), a quantification of the analyte could be possible as the detected assay signal can unequivocally be attributed to a defined amount of the analyte in the sample.

In case of one-step bridging assays, the standard assay format used for immunogenicity testing, the situation is special. On the one hand, capture and detection reagent are identical, as the drug is used as capture and detection reagent (bearing different labels), and thus only one bridging binding event is critical for the assay readout. On the other hand, however, the difficulty is the fact, that not one defined analyte is detected but a variety of analytes, namely the immune globulins against the drug, generated in the course of a polyclonal immune response. This means that a variety of immunoglobulin isotypes with different affinities are the actual analytes [9]. The typically used bridging assays enable the detection of all isotypes, as requested by the guidelines [4,5]. However, since the analytes, the generated human immunoglobulins, might show different binding properties as the used positive control antibody, a correct quantification is deemed not possible, but only a quasi-quantification, as already described by Mire-Sluis et al. in 2004 [13]. The lack of a true reference standard is also the argument why a correct sensitivity of an assay cannot be determined and that allegedly, the reported assay sensitivity depends on the used positive control.

Thus, on first sight, it is correct, that the use of different positive controls might result in different assay sensitivities, in other words, different amounts of the control are required to generate an assay signal at the defined cut-point level, leading to an 'apparent assay sensitivity' only. In an LBA, the signal is not directly generated by the positive control but by the amount of complexes formed between the analyte and the assay reagents. Still, based on the affinity of the ADA, it can be deduced at what concentrations of the reagents the maximum concentration of complexes is formed.

Therefore, in case of an ADA-bridging LBA, the actual critical parameter is the amount of 'signal-generating' ADA (positive control)-reagent complexes (ARCs) which are formed in solution during the incubation step (Figure 1). In case of a one-step bridging assay format, the amount of ARCs is dependent on: the amount of the ADA (positive control), the amount and ratio of the capture and detection reagents and the affinity between the ADA-positive control and the reagents.

The ADA assay sensitivity is investigated by analyzing a panel of quality control samples (QC) fortified with increasing concentrations of the ADA-positive control, to determine the concentration that generates a signal at the defined cut-point signal $[4,5]$.

Consequently, the amount of ADA (positive control) which generates a cut-point signal is known, as well as the amount of the assay reagents. The only missing piece of information is the affinity between the ADA-positive control and the reagents. The use of a positive control with characterized binding properties would easily enable 


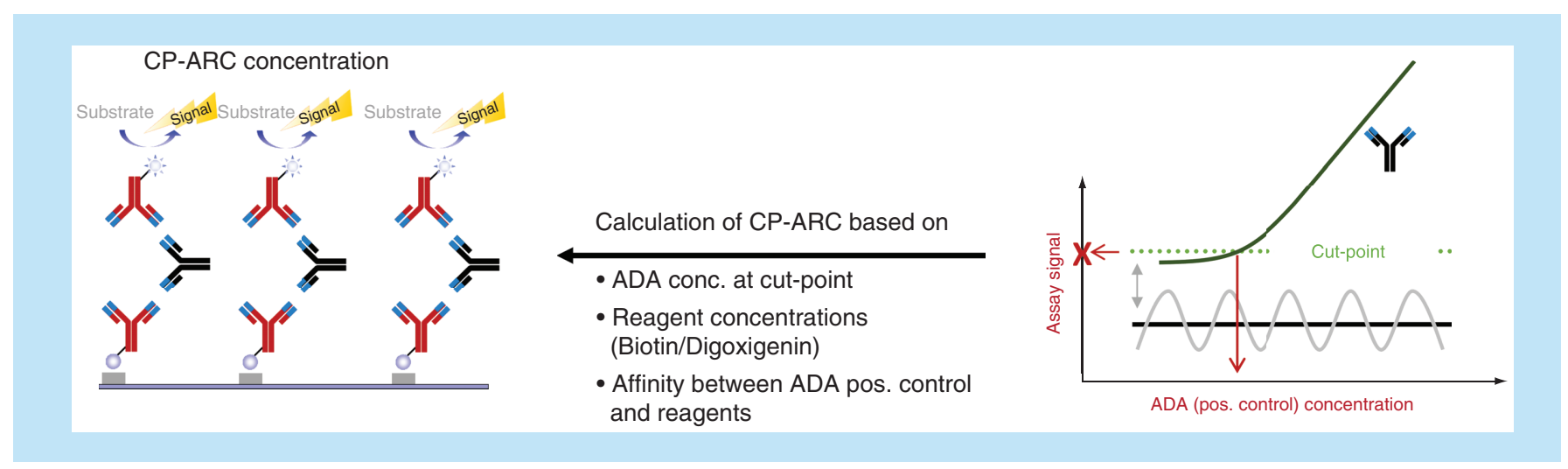

Figure 2. Cut-point-antibody reagent complex determination. CP-ARC is the amount of signal generating ARCs required to give an assay signal at the defined cut-point. CP-ARC is a constant assay performance parameter that defines the assay sensitivity and drug tolerance and enables assay comparisons. It can be determined by evaluating the amount of the ADA-positive control with known binding properties to the assay reagents that is required to give a signal at the defined cut-point followed by calculating the amount of complexes taking into consideration the used assay reagent concentrations as illustrated in Figure 1.

ADA: Anti-drug antibodies; CP-ARC: Cut-point anti-drug antibody-reagent complex.

the calculation of the amount of the 'cut-point ADA (positive control)-reagent complexes' (CP-ARC; Figure 2). This CP-ARC amount is an unequivocal parameter that defines when a positive signal is generated in a given assay, and thus when a sample is assessed as ADA positive. It goes without saying, that any changes of the assay (e.g., new reagent batches) that might affect the performance of the assay will also result in a change of the assay-specific CP-ARC.

The CP-ARC is a constant assay performance parameter. It can be used for the determination of the assay sensitivity at the defined cut-point independent of the positive control, since it allows evaluation of the true assay sensitivity range as a function of the ADA affinity. Knowledge of the CP-ARC amount is also an important parameter for the characterization of the drug tolerance of an ADA assay. The residual drug in the sample interferes with the assay by competing with the assay reagents, which are actually the differentially labeled drug, for binding with the ADA (positive control) and thus for formation of measurable complexes. This means that the residual drug is simply another binding partner in the equilibrium reaction. By implementing the amount of the residual drug in the sample into the calculation of the formation of signal generating ARCs, it can be evaluated at which drug-ADA-reagent concentration ratio a positive assay signal will be generated. Only those samples will generate a positive signal, in which at least the amount of CP-ARCs are formed.

\section{Determination of CP-ARC}

In case of a one-step ADA bridging assay, the binding of the capturing and the detection reagent happens simultaneously. Furthermore, the analyte (ADA or ADA-positive control) is a bivalent molecule, which results in the formation of mixed complexes that needs to be taken into consideration for the determination of the CP-ARC. Due to the bivalency of the ADA/ADA-positive control and the use of differently labeled reagents (e.g., biotin and digoxigenin), a mixture of analyte reagent complexes is formed: biotin/digoxigenin, biotin/biotin and digoxigenin/digoxigenin. This distribution of the formed complexes needs to be factored in, to determine the CP-ARC concentration, which is defined as the amount of 'correct' (i.e., biotin/digoxigenin) and thus signal generating ARCs which causes a signal at cut-point level.

In case of the presence of residual drug, additional non-signal generating complexes are formed. Besides the above-mentioned complexes, the ADA/ADA-positive control forms the following complexes: biotin/residual drug, digoxigenin/residual drug and residual drug/residual drug. All these complexes do not result in an assay signal and are the reason for the reduced ADA detectability in the presence of residual drug (drug tolerance; Figure 3).

For the evaluation at which ADA/ADA-positive control concentration a positive signal is generated in the presence of residual drug, the drug and consequently the additional complex formations have to be included into the calculation. It is obvious that these additional binding reactions of the ADA/ADA-positive control shift the equilibrium away from the formation of the correct (biotin/digoxigenin) complexes and thus the CP-ARC concentration is only formed at higher ADA concentrations. 


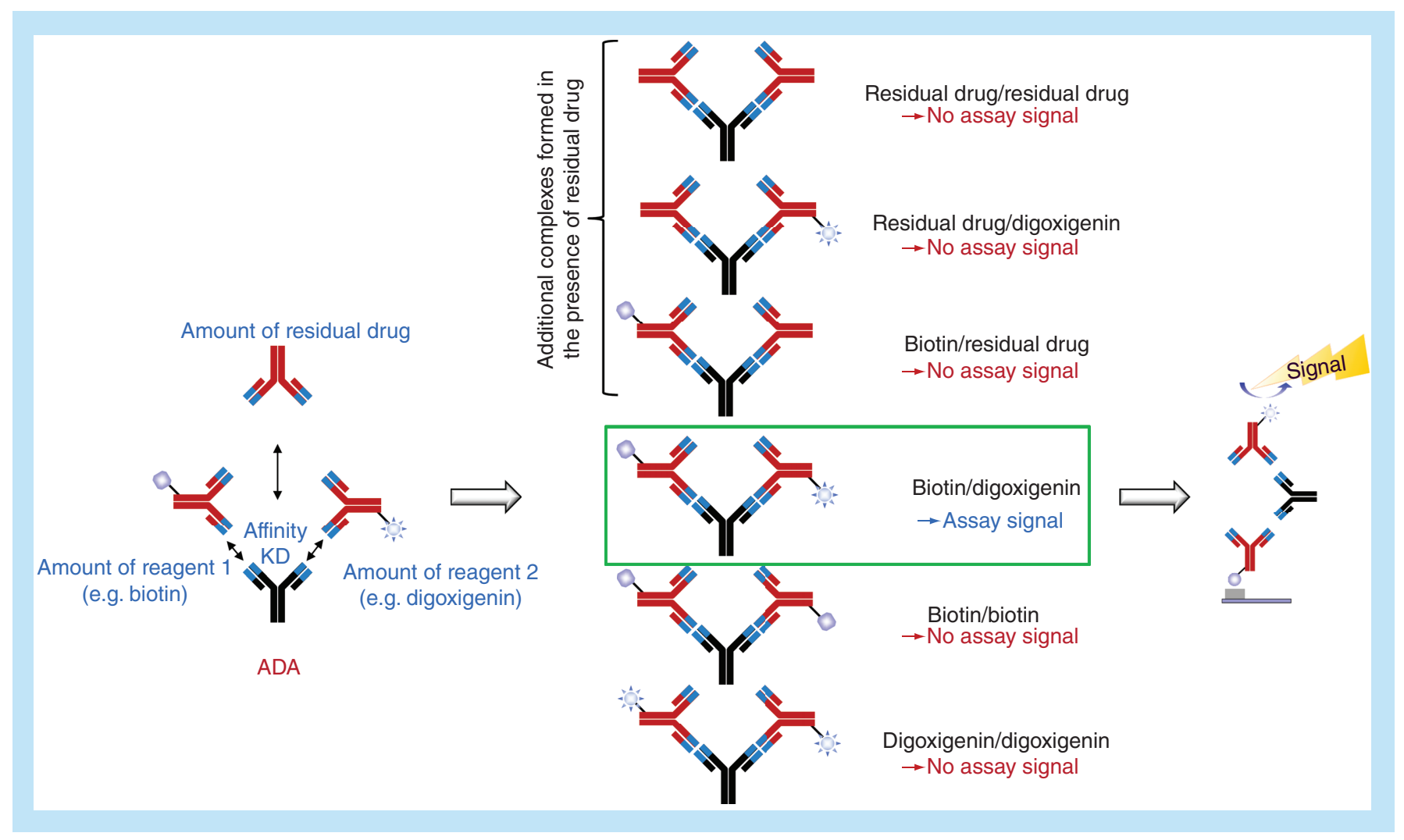

Figure 3. Drug tolerance. The presence of residual drug, another binding partner in the equilibrium reaction, leads to the formation of additional ADA-reagent/drug complexes that do not result in an assay signal and shift the equilibrium away from the formation of CP-ARCs. Consequently, lower assay signals are detected at an identical ADA concentration in the absence and presence of residual dug. By knowing the assay CP-ARC, the detectability of ADAs with varying affinities can clearly be calculated by integrating the residual drug concentration into the calculation of the equilibrium (1. ADA 2. Residual drug, 3. Reagents 4. Affinity) and by knowing the assay CP-ARC, the detectability of ADAs with varying affinities and consequently the drug tolerance of the assay can unequivocally be described. ADA: Anti-drug antibody; CP-ARC: Cut-point anti-drug antibody-reagent complex.

Is the CP-ARC hypothesis only theory? A case example

For the evaluation whether the CP-ARC hypothesis is only a theoretical model or applicable to real ADA assays, a conventional bridging ADA ELISA assay (minimal required dilution [MRD] of 100) with a dissociation step, using biotinylated and digoxigenylated monoclonal antibody drug at a concentration of $1000 \mathrm{ng} / \mathrm{ml}$ was used. The ADA-positive control was an immunoaffinity purified rabbit-derived polyclonal antibody preparation, with an affinity/KD of $0.1 \mathrm{nM}$ toward the drug/reagents.

In a first step, the CP-ARC of the assay was determined, by constructing a calibration curve with the ADApositive control ranging from 15.6 to $4000 \mathrm{ng} / \mathrm{ml}$ and by plotting the ADA-positive control concentration against the signal-to-blank ratio $(\mathrm{S} / \mathrm{B})$.

To keep it simple in this example, cut-points were defined at different S/B values $(2,6,20)$. The ADA concentration required to generate a signal at the respective S/B was determined using the calibration curve. This $\mathrm{ADA}$ (positive control) concentration, together with the known concentration of the reagents and the affinity of the ADA-positive control toward the reagents were used for the calculation of the respective CP-ARC concentration (1.5 pM, $7.7 \mathrm{pM}, 37.7 \mathrm{pM}$ at S/B 2, 6, 20, respectively). In a second step, a drug tolerance checkerboard experiment was performed, with the ADA-positive control ranging from 15.6 to $4000 \mathrm{ng} / \mathrm{ml}$ fortified with increasing drug concentrations up to $1000 \mu \mathrm{g} / \mathrm{ml}$.

For each residual drug concentration, the ADA calibration curves were plotted against the $\mathrm{S} / \mathrm{B}$ value, to determine the concentration of ADA-positive control to generate a positive signal (at $S / B=2,6$ or 20). Additionally, for each ADA-positive control/drug/reagents combination, the amount of CP-ARCs was calculated to evaluate at which ratio sufficient $\mathrm{ARCs}$ are formed to reach the $\mathrm{CP}-\mathrm{ARC}$ concentration resulting in the generation of a positive 


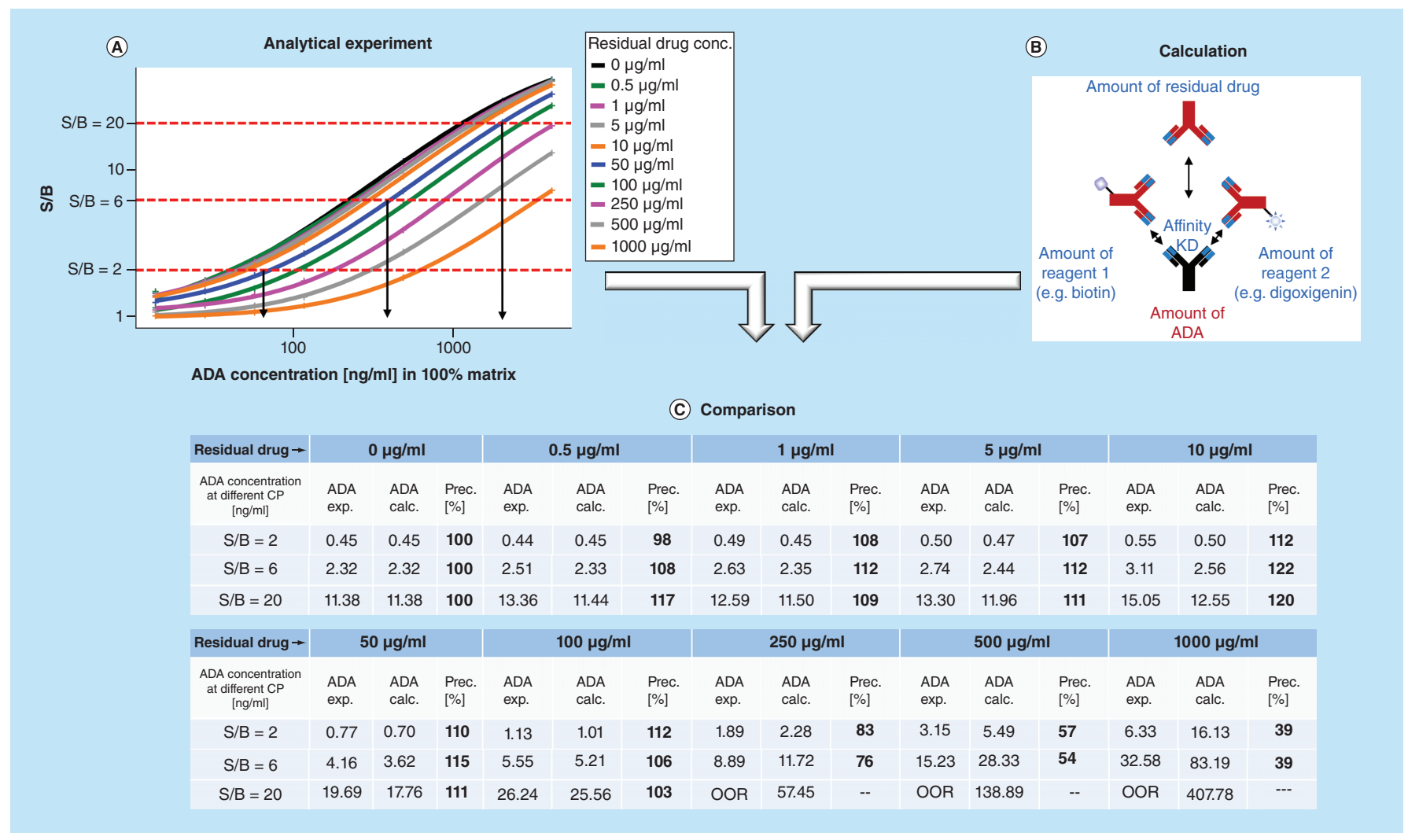

Figure 4. Comparison of experimentally determined and calculated CP-ARC concentrations. (A) Analytical experiment: the ADA concentrations that generated a positive assay signal in the presence of increasing residual drug concentrations $(0-1000 \mu \mathrm{g} / \mathrm{ml})$ at three different cut-points ( $S / B=2,6$ and 20 ) were experimentally determined. Additionally, the ADA concentrations (incorporating the minimal required dilution) required to form sufficient amounts of CP-ARCs were calculated for the identical conditions (B) and the precision of the calculated results in comparison of the experimentally determined concentration was evaluated (C).

ADA: Anti-drug antibody; CP-ARC: Cut-point anti-drug antibody-reagent complex.

signal (at $S / B=2,6$ or 20). Finally, the experimentally determined ADA-positive control concentration required to achieve a cut-point signal was compared with the calculated ADA concentration. The exercise was performed at three different cut-point levels $(S / B=2,6$ and 20) to cover the entire tested drug tolerance concentration range for evaluating whether the predictions are also possible at a much higher assay range and thus to gain more confidence in the hypothesis.

As shown in Figure 4-a very good correlation of the experimentally determined and the calculated concentrations of the ADA-positive control required to generate a positive signal was observed. Up to a residual drug concentration of $250 \mu \mathrm{g} / \mathrm{ml}$ the difference was below $25 \%$ (for cut-point at $\mathrm{S} / \mathrm{B}=2 \& 6$, at $\mathrm{S} / \mathrm{B}=20$ only residual drug concentrations up to $100 \mu \mathrm{g} / \mathrm{ml}$ could be evaluated). In our experience, the drug concentration in samples collected for ADA testing (typically at $\mathrm{C}_{\text {trough }}$ concentrations) usually contain residual drug concentrations below $250 \mu \mathrm{g} / \mathrm{ml}$. Only at very high residual drug concentrations the difference between experimentally determined versus calculated values were higher than $25 \%$, but still in the precision range of what would be achievable using a titer evaluation with a dilution factor of 2 .

This data indicate the correctness of the CP-ARC hypothesis and that this approach could successfully be applied to calculate and predict the drug tolerance of an assay.

\section{Challenges of the CP-ARC determination}

The determination of the CP-ARC requires knowledge of the amount of the reagents, the concentration of the ADA-positive control at cut-point level and the binding kinetics of the interaction of the ADA-positive control with the drug or the reagents. The affinity between the reagents (labeled drug) and the unlabeled drug should ideally be identical with no impact of the labeling on the binding. While the concentration of the reagents are known and the 
concentration of the ADA-positive control, which is required to generate a signal at the defined cut-point can easily be determined by constructing a calibration curve (Figure 2), the typically missing parameter is the information of the binding kinetics between ADA-positive control and the reagents. Thus, the additional effort necessary is the characterization of the binding properties of the ADA-positive control. For an appropriate calculation and description of the interaction, the in-solution binding kinetic information is required. Mathematical description of the interaction of binding partners by respective differential equations is a routine exercise in the field of modelling and simulation and even applied to better understand the impact of immunogenicity [25].

The feasibility of accurate calculation of in-solution interactions was already successfully shown in the field of free/active drug quantification even for bifunctional monoclonal antibodies [26-28]. The challenges encountered for the free QC concept are also valid for the calculation of the CP-ARC concentration. The most critical parameter is an accurate understanding of the in-solution interaction. In our experience, the affinity and binding kinetics parameter derived by an assay set up, where one of the binding partners is immobilized on a surface, for example, when a conventional Biacore experiment set-up is done, might be misleading. It is advisable to use an appropriate assay set up for the determination of the in-solution interaction to have binding kinetic data that really describe the interaction during the incubation of the sample with the reagents [29]. From a technological perspective the use of monoclonal antibodies as a positive control, seem to be favorable, since they allow an easier characterization of the binding interactions.

A prerequisite for a correct calculation is that an equilibrium between the different binding partners (ADA [positive control]), drug-biotin, drug-digoxigenin, residual drug) is reached. This can be accomplished by a sufficient incubation time, to assure dissociation of the existing ADA-drug complexes (potentially with different dissociation rates [koff]) and formation of the new complexes with the reagents, or by employing a dissociation step of the present ADA-drug complexes, which significantly speeds up the process. If a dissociation step is used, the time limiting step is the formation of the new complexes. This association (defined by the association rate constant [kon]) step is typically much faster than the dissociation of existing ADA-drug complexes and the new equilibrium is reached within the typical incubation times used for ADA assays.

\section{The benefits of the CP-ARC approach}

A detailed evaluation of the assay specific CP-ARC concentration significantly increases the understanding of an ADA assay and finally of the assay result interpretation. In the field of assay development, the knowledge of the CP-ARC concentration and the knowledge of the mutual interaction between ADA (positive control), reagents and residual drug, can be used to predict the drug tolerance of the assay. The CP-ARC concentration much better describes the assay performance, particularly with regard to the assay sensitivity, than the current state-of-the art process. By using the CP-ARC approach, a sensitivity range depending on the affinity of the ADA could be provided. This information is helpful to assess the assay performance independent of the used ADA-positive control.

To illustrate this, let us do a theoretical consideration and let us assume a bridging assay (MRD $=100$ and reagent concentrations $1000 \mathrm{ng} / \mathrm{ml}$, each) which enables the detection of an $\mathrm{ADA}$ (with an $\mathrm{KD}=0.1 \mathrm{nM}$ ) at a cut-point at $100 \mathrm{ng} / \mathrm{ml}$, as recommended by the FDA guideline [5]. Based on this information the respective amount of CP-ARCs could be calculated. If an ADA-positive control with a 100 -fold lower affinity $(10 \mathrm{nM})$ is used, $230 \mathrm{ng} / \mathrm{ml}$ would be needed to generate the CP-ARC concentration $(3 \mathrm{pM})$ to give a signal at cut-point level. If an ADA-positive control with a 100-fold higher affinity $(0.001 \mathrm{nM})$ would be used, $97 \mathrm{ng} / \mathrm{ml}$ would be needed. If only the low affinity ADA-positive control would have been available, one might wrongly have concluded that the assay is not 'sensitive enough', albeit it actually is. With the CP-ARC approach, any ADA-positive control with well-characterized binding properties could be used, independent whether the apparent sensitivity is below or above the recommended $100 \mathrm{ng} / \mathrm{ml}$ [5], since the concentration of the CP-ARC is the actual assay performance parameter.

Furthermore, this example shows that even a 100-fold lower affinity of the ADAs only results in a 2.3-fold lower sensitivity. A 100-fold higher affinity actually does not really increase the sensitivity (100 vs $97 \mathrm{ng} / \mathrm{ml}$ ). This theoretical exercise nicely shows the non-linear dependency between affinity of the analyte to the reagents and indicates that at a given affinity, further affinity increases do not increase the apparent assay sensitivity (in other words, already at $\mathrm{KD}=0.1 \mathrm{nM}$ nearly all $\mathrm{ADA}$ is bound to the reagents). It must be noted, that the correlation between ADA affinity and apparent assay sensitivity, is assay specific. However, with the knowledge of the CP-ARC concentration this information can easily be generated and a sensitivity range could be reported, for instance, in this example: sensitivity ranges from $230 \mathrm{ng} / \mathrm{ml}$ at $\mathrm{KD}=10 \mathrm{nM}$ to $97 \mathrm{ng} / \mathrm{ml}$ at $\mathrm{KD}=0.001 \mathrm{nM}$ ). 
To fully exploit the potential of this technical information, a better understanding of the affinity ranges of human ADAs would be helpful. Given that ADA assays have a sensitivity range, rather than a defined limit of detection, and that the CP-ARC approach enables an evaluation of what kind of ADAs (with regard to affinity) would be detectable even in the presence of residual drug, we could also improve the reporting of ADAs. In case of a negative ADA result in the presence of residual drug, Shankar et al. propose to use the term 'inconclusive' [8]. By using the CP-ARC approach, together with the knowledge of total drug concentration in the sample, a clear statement on what kind of ADAs would have been detectable could be given. Consequently, a kind of cut-off could be provided, for example, $<100 \mathrm{ng} / \mathrm{ml}$ ADA with an affinity of xyz $\mathrm{nM}$, a much better defined information for further decision making, which would significantly improve the quality of the bioanalytical data package.

With the use of well-characterized ADA-positive controls and the knowledge that the CP-ARC as the actual constant assay sensitivity parameter an alternative cut-point determination approach would be conceivable. The current cut-point determination based on analysis of a defined panel of blank samples [4-6,13] significantly suffers from the fact, that blank samples only show low signals close to instrument noise in high quality ADA assays [30,31]. As a consequence the statistical evaluation of the cut-point is done on instrument noise instead of real signals. Similar to the preparation of free QCs [26], where, based on the affinity, defined amounts of drug and target are mixed to form defined amounts of free drug and drug-ligand complexes at equilibrium, samples of defined ARCs could be generated by mixing ADA-positive control with the ADA assay reagents. A kind of ARC calibration curve could be generated, to enable a 'top-down' cut-point approach similar to the evaluation of assay sensitivity in a PK assay. The huge benefit would be that the statistical evaluation to achieve the desired level of false positivity and to define the assay sensitivity as the CP-ARC concentration could be based on real assay signals instead of instrument noise.

\section{Conclusion}

An important step in the field of immunogenicity testing would be the possibility to compare clinical immunogenicity observed for different compounds. A prerequisite to reach this goal is to achieve comparability of the technical performances of the bioanalytical methods used for immunogenicity testing. The typically used bridging LBAs for the detection of ADA and consequently to define the immunogenicity incidence of a therapeutic protein, are considered non-comparable. The critical assay performance parameters are different assay sensitivities and different drug tolerances, which are particularly important since in most cases immunogenicity testing is performed using study samples that still contain considerable residual drug concentrations.

Currently, the sensitivity and drug tolerance is evaluated during assay validation using an ADA-positive control antibody. For this purpose, animal derived immunopurified polyclonal antibody preparations or monoclonal antibodies are recommended. Sensitivity is reported as the concentration of the ADA-positive control required to generate an assay signal that was defined as cut-point. Drug tolerance is evaluated in a checkerboard experiment where increasing ADA-positive control concentrations are fortified with increasing drug concentration to evaluate at what drug concentration what ADA-positive control concentration still gives a positive assay signal. The results of these experiments, however, are unfortunately limited and have to be considered only as apparent sensitivity or drug tolerance. For LBAs, the binding of the analyte and the reagents is the most critical step. For ADA testing, this is particularly challenging since the analyte is not one defined compound with a defined binding reaction but immunoglobulins of a polyclonal immune response with different binding properties have to be analyzed. If the affinity of the used positive control is different from the affinity of the real ADAs, even at the same concentration, different assay signals will be generated and thus the assay result is not comparable.

To overcome this challenge, we need to better evaluate the actual critical step of the assay, the binding of the reagents to the analyte, which finally results in the formation of ADA-reagent complexes. The focus needs to be on the formation of the assay signal generating complexes, which we titled as ARCs. As shown by the given example, the amount of ARCs required to generate a positive assay signal (CP-ARC), is a constant, clearly defined assay parameter that is independent from the affinity of the used ADA-positive control. Furthermore, the comparison of the CP-ARC values determined for different assays could allow an improved comparison between different LBA conditions, independent of the used positive control and thus might enable immunogenicity data comparison independent of the used bioanalytical method/technology.

The determination of the CP-ARC only requires the full scientific exploitation of the data that is generated anyhow during assay development and the use an ADA-positive control with characterized binding properties, 
particularly the affinity $(\mathrm{KD})$. With known reagent concentrations, affinity and the determined concentration of the ADA-positive control at the cut-point, the CP-ARC concentration can easily be determined.

Thus, the efforts required for the determination of the CP-ARC are manageable and should be feasible during any assay validation. With the knowledge of the CP-ARC a much better description of the assay performance is possible. A real assay sensitivity range, depending on the affinity range of the ADAs, could be determined as well as the drug tolerance. In case of a negative assay result, a clear statement could be provided what affinity and concentration range of ADAs would have been detectable at the given residual drug concentration in the sample. Thus, instead of providing 'inconclusive' data results, clear statements would be possible.

Since the CP-ARC concept is independent on the used positive control, an improved scientific description of the actual assay performance could be provided. This, in turn, enables a much better and even technology independent comparison of the assay performances of different assays, which again allows comparison of the bioanalytical data and finally helps to achieve the goal to be able to compare the clinical immunogenicity of different compounds. This would significantly help to enable a rational internal project decision making, would enable the regulators to better asses the submitted immunogenicity data package and finally would enable the selection the best treatment for the patients by physicians.

\section{Future perspective}

A joint effort of the entire bioanalytical community is key to achieve the ultimate goal of comparability of the clinical immunogenicity of different compounds. We should take the responsibility to find scientific solutions to provide assays and finally data packages that are clearly defined and enable a comparison. Since a critical look at our current processes is required and new ways and experiments have to be implemented, a truly joint effort of the whole community is needed, from the assay developers to the regulators. Only such a joint effort enables an in-depth scientific discussion which might result in a consensus and a definition of what data is required for comparability of immunogenicity data.

With the proposal of the CP-ARC concept, we would like to initiate the discussion, focusing on one-step bridging assays since this is the gold standard format for immunogenicity testing. We consider this approach as a very promising and easily implementable into our current bioanalytical processes. The only additional effort is to characterize the in-solution binding properties of the used ADA-positive control, including the discussion whether monoclonal or polyclonal antibody preparations are the better ADA-positive control. Concerning the characterization of the in-solution binding properties, the use of monoclonal ADA-positive controls is less complicated and thus favorable. Since this task is in many cases not the responsibility of the bioanalytical groups and since the in-solution binding kinetics might significantly differ from what is observed in conventional binding evaluations with binding partners immobilized onto a surface, a discussion with the respective experts is required. Ideally, fully characterized ADA-positive control antibodies will be available for every ADA assay development and the determination of the CP-ARC concentration becomes a routine task.

Further discussions are necessary on how the assay performance parameters (sensitivity range - drug tolerance range) should be reported, to enable an easy comparison. This proposal is focused on the technical performance of the assays. The next step is to expand the increased knowledge of what information our assays provide to the interpretation of $\mathrm{ADA}$ positive sample results. A better understanding of the in-solution binding properties of real human ADAs would be a highly desirable goal, which needs scientific investment. Another furture step is the detailed evaluation of potential avidity binding and inclusion into the CP-ARC concept to also expand this concept to heterogeneous assay formats (e.g., use drug-coated surfaces for a capture assay format) or technology specific peculiarities in case of, for example, SPR were the dissociation of the formed CP-ARC from the measurement surface has to be incorporated into the model.

Ideally, after solutions to the open questions have been found, the bioanalytical data package to support immunogenicity testing could be complemented by ADA affinity characterization. With this missing piece, a definitive statement on the ADA positivity or negativity of a sample, even in the presence of residual drug, will be possible. Furthermore, knowledge of the binding properties of real human ADAs might open new possibilities for true ADA quantification.

\section{Acknowledgments}

The authors thank M Zaspel for technical support and J Heinrich for scientific discussion. 
Executive summary

- Clinical immunogenicity testing is a well-defined and established process of clinical drug development, but still lacks comparability of results of different assays and compounds.

- The cut-point anti-drug antibodies-reagent complexes (CP-ARC) concept is proposed to enable comparability of ADA assays, independent of the used ADA-positive control and independent of the applied LBA technology.

- The CP-ARC, the amount of ADA-reagent complexes required to generate a signal at cut-point level in a given assay, is a constant assay parameter that enables a clear understanding of the true assay sensitivity range and drug tolerance.

- The CP-ARC concept could easily be implemented into routine ADA assay development/validation, since it is based on available information (concentration of the ADA-positive control and assay reagents) and the affinity between the used positive control and the reagents that could be generated with little effort.

- The benefit of implementation of the CP-ARC concept is:

- A clear understanding of the assay performance with regard to sensitivity and drug tolerance.

- An improved reporting of bioanalytical immunogenicity results.

- Comparability of bioanalytical assay results that finally enables comparability of the clinical immunogenicity of different compounds.

Financial \& competing interests disclosure

The authors are employees of Roche Diagnostics $\mathrm{GmbH}$ and are owners of Roche bonus shares and profit certificates. The authors have no other relevant affiliations or financial involvement with any organization or entity with a financial interest in or financial conflict with the subject matter or materials discussed in the manuscript apart from those disclosed.

No writing assistance was utilized in the production of this manuscript.

\section{Open access}

This work is licensed under the Attribution-NonCommercial-NoDerivatives 4.0 Unported License. To view a copy of this license, visit http://creativecommons.org/licenses/by-nc-nd/4.0/

\section{References}

Papers of special note have been highlighted as: $\bullet$ of interest; $\bullet \bullet$ of considerable interest

1. Chirmule N, Jawa V, Meibohm B. Immunogenicity to therapeutic proteins: impact on PK/PD and efficacy. AAPS J. 14(2), 296-302 (2012).

2. Schellekens H. Immunogenicity of therapeutic proteins: clinical implications and future prospects. Clin. Ther. 24(11), 1720-1740 (2002).

3. Smith A, Manoli H, Jaw S et al. Unraveling the effect of immunogenicity on the PK/PD, efficacy, and safety of therapeutic proteins. J. Immunol. Res. 2016, 2342187 (2016).

4. European Medicines Agency. Guideline on immunogenicity assessment of therapeutic proteins (2017). www.ema.europa.eu/en/docum ents/scientific-guideline/guideline-immunogenicity-assessment-therapeutic-proteins-revision-1_en.pdf

5. U.S. Department of Health and Human Services Food and Drug Administration Center for Drug Evaluation and Research (CDER) Center for Biologics Evaluation and Research (CBER). U.S. Food and Drug Administration; Immunogenicity Testing of Therapeutic Protein Products - Developing and Validating Assays for Anti-Drug Antibody Detection Guidance for Industry. Guidance for Industry (2019). www.fda.gov/regulatory-information/search-fda-guidance-documents/immunogenicity-testing-therapeutic-protein-products-d eveloping-and-validating-assays-anti-drug

6. Devanarayan V, Smith WC, Brunelle RL, Seger ME, Krug K, Bowsher RR. Recommendations for systematic statistical computation of immunogenicity cut points. AAPS.J. 19(5), 1487-1498 (2017).

7. Shankar G, Devanarayan V, Amaravadi L et al. Recommendations for the validation of immunoassays used for detection of host antibodies against biotechnology products. J. Pharm. Biomed. Anal. 48(5), 1267-1281 (2008).

8. Shankar G, Arkin S, Cocea L et al. Assessment and reporting of the clinical immunogenicity of therapeutic proteins and peptides-harmonized terminology and tactical recommendations. AAPS J. 16(4), 658-673 (2014).

9. Gunn GR III, Sealey DC, Jamali F, Meibohm B, Ghosh S, Shankar G. From the bench to clinical practice: understanding the challenges and uncertainties in immunogenicity testing for biopharmaceuticals. Clin. Exp. Immunol. 184(2), 137-146 (2016).

10. Civoli F, Kasinath A, Cai XY et al. Recommendations for the development and validation of immunogenicity assays in support of biosimilar programs. AAPS J. 22(1), 7- (2019).

11. Kim JS, Kim SH, Kwon B, Hong S. Comparison of immunogenicity test methods used in clinical studies of infliximab and its biosimilar (CT-P13). Expert. Rev. Clin. Immunol. 11(Suppl. 1), S33-S41 (2015). 
12. Ryding J, Stahl M, Ullmann M. Demonstrating biosimilar and originator antidrug antibody binding comparability in antidrug antibody assays: a practical approach. Bioanalysis. 9(18), 1395-1406 (2017).

13. Mire-Sluis AR, Barrett YC, Devanarayan V et al. Recommendations for the design and optimization of immunoassays used in the detection of host antibodies against biotechnology products. J. Immunol. Methods 289(1-2), 1-16 (2004).

14. Egging D, Verhagen J, Laat-Arts $\mathrm{K}$ et al. Sensitivity and drug tolerance of antidrug antibody assays in relation to positive control characteristics. Bioanalysis 10(16), 1289-1306 (2018).

15. Ishii-Watabe A, Shibata $\mathrm{H}$, Nishimura $\mathrm{K}$ et al. Immunogenicity of therapeutic protein products: current considerations for anti-drug antibody assay in Japan. Bioanalysis 10(2), 95-105 (2018).

16. Li J, Schantz A, Schwegler M, Shankar G. Detection of low-affinity anti-drug antibodies and improved drug tolerance in immunogenicity testing by $\operatorname{Octet}((\mathrm{R}))$ biolayer interferometry. J. Pharm. Biomed. Anal. 54(2), 286-294 (2011).

17. Liang M, Klakamp SL, Funelas $\mathrm{C}$ et al. Detection of high- and low-affinity antibodies against a human monoclonal antibody using various technology platforms. Assay Drug Dev. Technol. 5(5), 655-662 (2007).

18. Shankar G, Pendley C, Stein KE. A risk-based bioanalytical strategy for the assessment of antibody immune responses against biological drugs. Nat. Biotechnol. 25(5), 555-561 (2007).

19. Gupta S, Richards S, Amaravadi L et al. 2017 White Paper on recent issues in bioanalysis: a global perspective on immunogenicity guidelines \& biomarker assay performance (Part 3 - LBA: immunogenicity, biomarkers and PK assays). Bioanalysis 9(24), 1967-1996 (2017).

20. Kontermann RE, Brinkmann U. Bispecific antibodies. Drug Discov. Today 20(7), 838-847 (2015).

21. Duo J, Bruno J, Kozhich A et al. Surface plasmon resonance as a tool for ligand-binding assay reagent characterization in bioanalysis of biotherapeutics. Bioanalysis 10(8), 559-576 (2018).

22. King LE, Farley E, Imazato $\mathrm{M}$ et al. Ligand binding assay critical reagents and their stability: recommendations and best practices from the Global Bioanalysis Consortium Harmonization Team. AAPS J. 16(3), 504-515 (2014).

23. O'Hara DM, Theobald V, Egan AC et al. Ligand binding assays in the 21 st century laboratory: recommendations for characterization and supply of critical reagents. AAPS J. 14(2), 316-328 (2012).

24. Staack RF, Stracke JO, Stubenrauch K, Vogel R, Schleypen J, Papadimitriou A. Quality requirements for critical assay reagents used in bioanalysis of therapeutic proteins: what bioanalysts should know about their reagents. Bioanalysis 3(5), 523-534 (2011).

25. Gomez-Mantilla JD, Troconiz IF, Parra-Guillen Z, Garrido MJ. Review on modeling anti-antibody responses to monoclonal antibodies. J. Pharmacokinet. Pharmacodyn. 41(5), 523-536 (2014).

\section{-. Very informative review on $M \& S$ of an immune repsonse.}

26. Staack RF, Jordan G, Dahl U, Heinrich J. Free analyte QC concept: a novel approach to prove correct quantification of free therapeutic protein drug/biomarker concentrations. Bioanalysis 6(4), 485-496 (2014).

27. Schick E, Staack RF, Haak M et al. Validation of a ligand-binding assay for active protein drug quantification following the 'free analyte QC concept'. Bioanalysis 8(24), 2537-2549 (2016).

28. Jordan G, Onami I, Heinrich J, Staack RF. Evaluation of the potential use of hybrid LC-MS/MS for active drug quantification applying the 'free analyte QC concept'. Bioanalysis 9(21), 1705-1717 (2017).

29. Drake AW, Tang ML, Papalia GA, Landes G, Haak-Frendscho M, Klakamp SL. Biacore surface matrix effects on the binding kinetics and affinity of an antigen/antibody complex. Anal. Biochem. 429(1), 58-69 (2012).

- Interessting work that shows the challenge to determine relevant affinity data.

30. Goodman J, Cowen S, Devanarayan V et al. Feedback from the European Bioanalysis Forum: focus workshop on current analysis of immunogenicity: best practices and regulatory hurdles. Bioanalysis 10(4), 197-204 (2018).

31. Myler H, Gorovits B, Phillips K et al. Report on the AAPS Immunogenicity Guidance Forum. AAPS.J. 21(4), 55 (2019). 
